\title{
Power Current Control of a Resonant Vibratory Conveyor Having Electromagnetic Drive
}

\author{
Željko V. Despotović†, Aleksandar I. Ribić ${ }^{*}$ and Vladimir M. Šinik ${ }^{* *}$ \\ ${ }^{\dagger *}$ Mihajlo Pupin Institute, University of the Belgrade, Serbia, \\ ** Technical Faculty “Mihajlo Pupin” Zrenjanin, University of the Novi Sad, Novi Sad, Serbia
}

\begin{abstract}
The vibratory conveyors with electromagnetic drive are used for performing gravimetric flow of granular materials in processing industry. By realizing free vibrations of variable intensity and frequency over a wide range through application of the electromagnetic actuator, suitable power converter, and the corresponding controller, continuous conveyance of granular materials have been provided for various operating conditions. Standard power output stages intended for control of vibratory conveyance using thyristors and triacs. Phase angle control can only accomplish tuning of amplitude oscillations, but oscillation frequency cannot be adjusted by these converters. Application of current controlled transistor converters enables accomplishing the amplitude and/or frequency control. Their use implies the excitation of a vibratory conveyor independent of the supply network frequency. In addition, the frequency control ensures operation in the region of mechanical resonance. Operation in this region is favourable from the energy point of view, since it requires minimal energy consumption. The paper presents a possible solution and advantages of the amplitude-frequency control of vibratory conveyors by means of a current controlled power converter.
\end{abstract}

Key words: Current control, IGBT, Power converter, Resonance, Vibrations control, Vibratory conveyor

\section{INTRODUCTION}

Vibratory movement is one of the most efficient methods of conveyance of granular and particulate materials. Vibratory conveyors are widely applied in many technological processes involving gravimetric transport, processing, and dosing of granular materials. From the macroscopic point of view, the process of vibratory conveyance is based on recurrent micro-throws of particles of the material being conveyed [1]-[4]. Vibrations of the load (vibratory trough), i.e. of the carrying element, the so called load carrying element (LCE), containing the material cause movement of particles of the material, therefore the material obtains the character of a viscous fluid and as such becomes suitable for conveyance, dosing, or further processing. The elastic connection of LCE to the carrying support is usually realized by tilted plate springs or by the springs made of

Manuscript received May 19, 2011; revised May 24, 2012

Recommended for publication by Associate Editor Tae-Woong Kim.

${ }^{\dagger}$ Corresponding Author: zeljko.despotovic@pupin.rs

Tel: +381-11-2771-024, Fax: +381-11-2776-583, Univ. of the Belgrade

** Technical Faculty "Mihajlo Pupin” Zrenjanin, University of the Novi Sad, Serbia

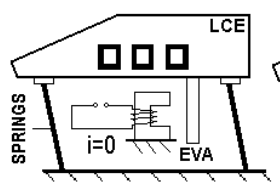

(a)

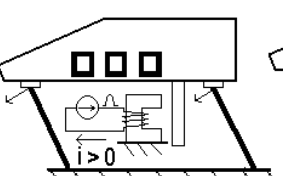

(b)

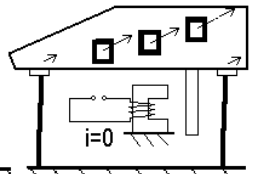

(c)
Fig. 1. The simplified representation of the vibratory conveyance. (a) standstill, (b) straining and (c) moving.

composite materials (fiberglass), as shown in Fig. 1.

The size and shape of the micro-throws are dependent upon the frequency and amplitude of vibrations of LCE. In recent times, the operation of these conveyance systems in the region of mechanical resonance has become very interesting since in this regime a relatively small energy is required for maintaining the system in the oscillatory state. Resonant vibration transporters having electromagnetic drives are used very often in these applications.

The driving element is in fact an electromagnetic vibratory actuator (EVA) which enables in various ways [1], [5] the incremental displacements of the LCE. Fig. 1 shows a process of conveyance of particles of the material being conveyed. The process is characterized by three phases. 
In phase (I) there is static equilibrium i.e. standstill. The excitation current through coil EVA is zero and the elastic springs of the system are strained only by the weight of the vibratory trough and the material it contains. The whole system is in the state of static equilibrium, i.e. in the initial position for starting to move. In phase (II) a current pulse of predefined amplitude and duration flows through coil EVA, armature EVA is pulled closer and the elastic springs are additionally strained. Work of the electromagnetic force EVA is converted to potential energy of the system which consists of the conveyance mass and elastic springs. The conveyed material is still on the LCE surface. In phase (III), current through EVA coil is discontinued and the accumulated potential energy is converted to the kinetic energy of the moving LCE, which is followed by displacement of the conveyed material. If the frequency of the excitation current pulses is tuned to a value close to the mechanical resonance of the system, it is possible to achieve high efficiency and accomplish significant vibratory energy of the conveyance, i.e. a high capacity of the conveyance, at the expense of a very small consumption of electrical energy. In addition, the absence of any group of moving parts (bearings, eccentrics, transmission belts, rotating parts, etc.) is of considerable significance as regards the minimization of the maintenance costs.

By a suitable control of the conveyor's excitation force, which is obtained by means of EVA, it is possible to accomplish both amplitude and frequency control of the vibratory conveyance. In this way the whole conveyance system behaves like a fully controlled mechanical oscillator. For most of the vibratory conveying drives of this type the range of amplitudes is from $0.1 \mathrm{~mm}$ up to $5 \mathrm{~mm}$ and the range of operating frequencies is from $5 \mathrm{~Hz}$ up to $150 \mathrm{~Hz}$, depending upon the type of the conveying material and load [6].

\section{POWER CONVERTER IN VIBRATORY CONVEYING}

Application of vibratory drives in combination with power converters provides a significant flexibility in fulfilling the requirements for conveyance of granular materials. At present, thyristors and triacs are used as standard semiconductor output power stages for driving electromagnetic vibratory conveyors. Their application implies adjustment of vibratory width (double amplitude of the oscillations) of the LCE by means of phase control, i.e. regulation of the phase angle $\alpha^{*}$ [7]-[10].

Basic topologies of the standard power converters, together with the corresponding waveforms of the EVA current and voltage, are shown in Figs. 2 and 3.

One type of these converters - unidirectional, having pulsating dc output current, makes use of only one half-period of the mains voltage. It is realized by using one thyristor, as shown in Fig. 2(a). In this type of converter the tyhristor is

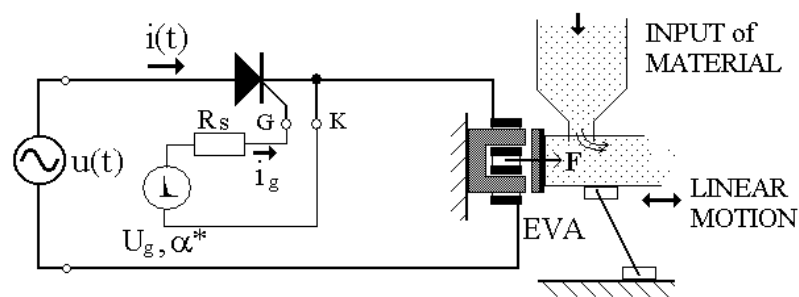

(a)

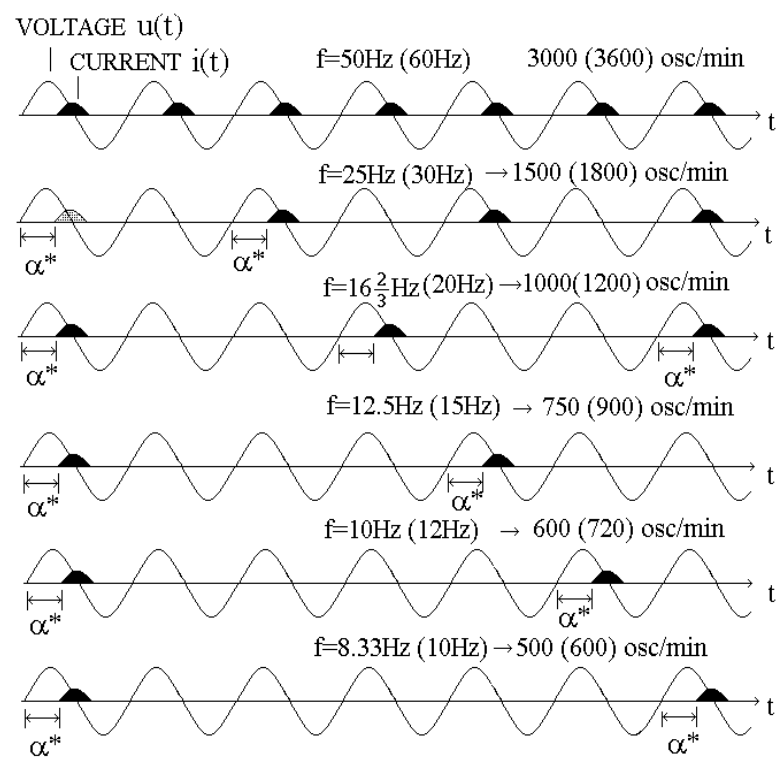

(b)

Fig. 2. Phase controlled thyristor converter for driving vibratory conveyors. (a) The unidirectional topology and (b) The waveforms of the EVA current and voltage.

triggered only during positive half-periods, as indicated in Fig. 2(b). In this way the mains voltage of frequency $50(60) \mathrm{Hz}$ at the input of the converter is converted to a pulsating dc current which supplies EVA coil. By applying this control it is possible o obtain a discrete frequency spectrum of the input current: $50(60) \mathrm{Hz}, \quad 25(30) \mathrm{Hz}, 16.66(20) \mathrm{Hz}, 12.5(15) \mathrm{Hz}, 10(12) \mathrm{Hz}$, $8.33(10) \mathrm{Hz}$, i.e. a spectrum of discrete vibrations of LCE: 3000(3600) cycles/min, 1500(1800) cycles/min, 1000(1200) cycles/min, 750(900) cycles/min, 600(720) cycles/min, 500(600) cycles/min.

Another type of converter-bidirectional or alternating output current converters, make use of both half-periods of the mains voltage. It is designed by using triacs and, by using anti-parallel connection of thyristors for high powers, as shown in Fig. 3(a). With this type of converter the mains voltage of frequency $50(60) \mathrm{Hz}$ is converted to an alternating current of the frequency which is the same as the one which supplies EVA coil, as shown in Fig. 3(b).

Since the excitation force of EVA coil is function of the 


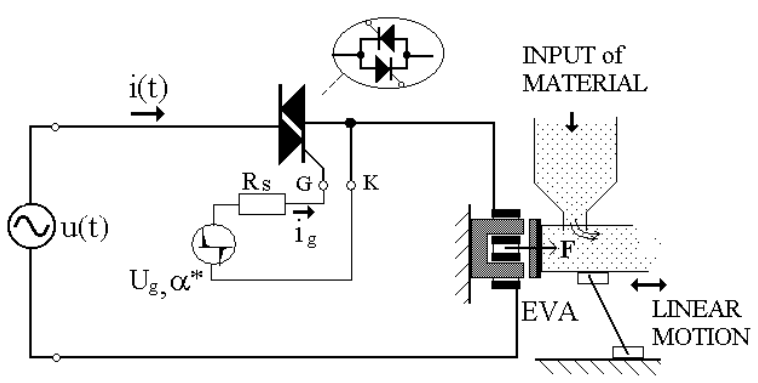

(a)

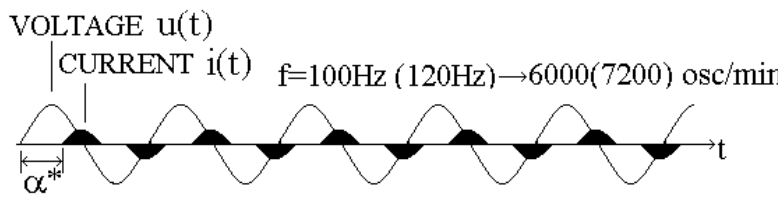

(b)

Fig. 3. Phase controlled thyristor converter for driving vibratory conveyors. (a) The bidirectional topology and (b)The waveforms of the EVA current and voltage.

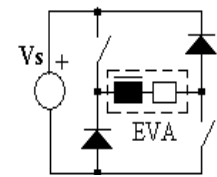

(a)

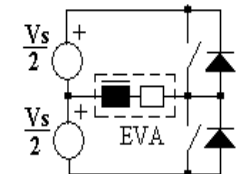

(b)

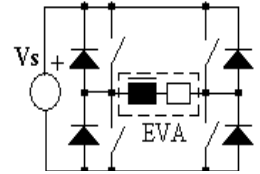

(c)
Fig. 4. Switching converter topologies for EVA excitation. (a) The asymmetric half-bridge, (b) The symmetric half-bridge and (c) The full-bridge.

square current flowing through the coil [9], [11], [12], with the first type of converters one generates excitation force of the maximum frequency $50(60) \mathrm{Hz}$ producing vibrations of 3000(3600) cycles/min, whereas with the second type of converter the maximum frequency of the excitation force is 100(120) Hz producing vibrations of 6000(7200) cycles/min.

Application of the phase controlled thyristor or triac converters in vibratory conveyance implies a fixed frequency of vibrations, imposed by the supply network frequency. A serious problem arises when the mass of the conveying material is changed, i.e. mechanical resonance of the system has changed. In such case the vibratory system will not operate efficiently. It is possible to tune amplitude but not the frequency of the vibrations. In addition, the thyristor converter brings in a dc component and undesirable higher harmonics. Application of triacs results in somewhat better situation as regards the harmonic content, but the same problem arises if the resonant frequency is changed. Variation of the mechanical resonance due to variation of the mass of the conveyed material, or even variation of the system parameters (characteristics of the springs, damping, etc.), leads to reduction of efficiency of the vibratory drives. In order to accomplish an optimal and efficient operation at a new resonant frequency, it is necessary to change the frequency of EVA supply current, i.e. of the excitation force of the vibratory conveyor.

The work on application of high-frequency (HF) transistor converters for obtaining sinusoidal current through EVA coil has been intensified recently. Like with thyristor converters, one can talk of unidirectional and bidirectional types, depending whether a pulsating dc current or an alternating excitation current is accomplished [9], [10], [12]. Mainly, the three topologies, shown in Fig. 4, have been accepted.

The topology consisting of two switches and two return diodes is used in designing the unidirectional type of converters, i.e. the asymmetric half-bridge, while the full-bridge and half-bridge topologies are used for designing the bidirectional type of converters. The required sine-wave (half-wave) can be realized by these topologies if the applied current control is based on tracking the reference sine-wave of adjustable length, amplitude, and frequency. This method of generation of the excitation current has the advantage in that it allows independent tuning of the frequency and amplitude of the electromagnetic excitation force $f$. The switching converter described in [9], despite its advantages, suffers from a serious shortcoming that at high frequencies its switching losses become dominant. In addition to these losses, the losses in iron of the magnetic circuit and in copper of EVA coil become also significant. This reduces the efficiency of the vibratory conveyor and it is not unusual that the power of losses in the system converter-EVA-vibratory drive is higher than the power required for maintaining the resonant oscillatory mode. This reduces considerably the efficiency of the drive as a whole. By a suitable control of the switches in these topologies, it is possible to overcome this problem and accomplish the expected vibratory effect, i.e. the required amplitude of LCE oscillations and optimal operating frequency of the vibratory conveyor.

\section{MATHEMATICAL MODEL OF THE VIBRATORY CONVEYOR WITH ELECTROMAGNETIC DRIVE}

A typical vibratory conveyor in a system of dosing granular material is shown in Fig. 5. It consists of a load carrying element ( $L C E$ ) -1, electromagnetic vibratory actuator - EVA as the source of the excitation force, and elastic elements -2 . Standard structure of these elements is lamellar, made of steel sheets. Recent practice is to use flexible elements made of composite materials (Fiberglass). These elements are fastened stiff to the base - 3, which rests on rubber supports - 4 elastically with respect to the surface supporting of the mechanism. EVA consists of: a magnetic core - 5, closed in an electrical coil - 6, and an armature - 7, which is fixed to a movable LCE, i.e. vibrating trough -8 along which the material is being conveyed. Electromagnetic driving force $f$ generated by EVA acts upon the armature, i.e. upon the vibrating trough. The vibratory displacement is detected by a 


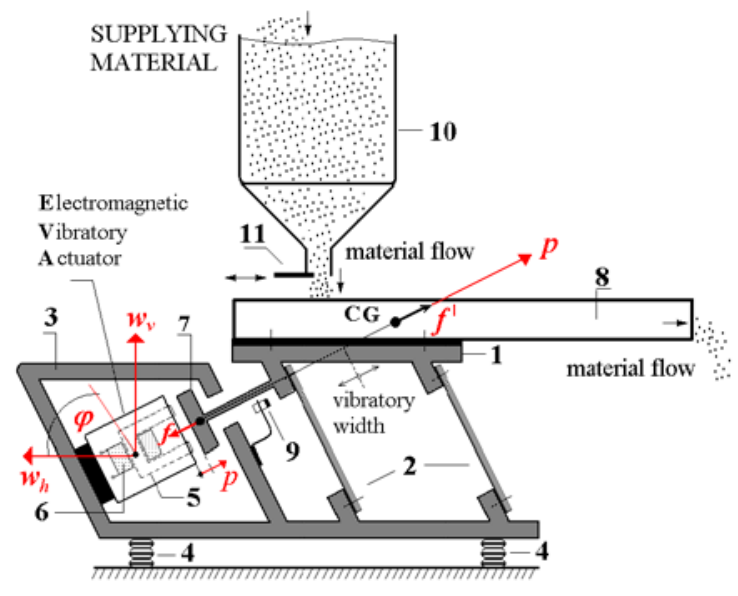

Fig. 5. Vibratory resonant conveyor having electromagnetic drive.

noncontact inductive sensor - 9. The material which is dosed is brought to the LCE from a storage hopper - 10. Adjustment of the material inflow is accomplished by a movable shutter 11 which is placed at the bottom of the hopper.

For defining control strategy of the vibratory conveyor, it is required to analyze the electromagnetic and mechanical parts of the system. Detailed dynamic model of the LCE supported by elastic springs, presented in [7], [8] and [13], [14], is of the form:

$$
M \cdot \ddot{z}+C \cdot \dot{z}+K \cdot z=\Gamma \cdot f
$$

Vector $\quad z \in R^{4} \wedge z=\left[\begin{array}{llll}p & w_{h} & w_{v} & \varphi\end{array}\right]^{T} \quad$ represents position vector and it contains the relative displacement - $p$ of the LCE with respect to the support, displacements of the base $-w_{h}, w_{v}$ in the horizontal and vertical directions respectively, and angle of inclination of the base- $\varphi$ with respect to its centre of gravity.

$M, \quad C$ and $K$ are the symmetric matrices. Vector $\Gamma=\left[\begin{array}{llll}-f & 0 & 0 & 0\end{array}\right]^{T}$ defines the excitation force acting on the LCE [7-8]. Equation (1) indicates that the mechanical system has four resonant modes. It should be mentioned that for the range of interesting vibrations, all four modes are not of interest. In practical applications the largest contribution to moving the LCE has a dominant oscillatory mode, coming from high quality composite springs and related to the displacement $p$ [7]:

$$
\ddot{p}+2 \xi \cdot \omega_{0} \dot{p}+\omega_{0}^{2}\left(p-p_{0}\right)=K_{p} \cdot \omega_{0}^{2} \cdot f
$$

where $\omega_{0}(\mathrm{rad} / \mathrm{s}), \xi$, and $K_{p}$ denote the resonant frequency, damping factor, and static gain, respectively.

The electromagnetic part is represented by the vibratory actuator EVA as the generator of the excitation force $f$ which is, through the electromagnetic field in the air gap between the inductor and armature, transferred to LCE. Detailed model of EVA, presented in [5] and [7], [8], can be represented in the form:

$$
\begin{gathered}
L(p) \cdot \frac{d i}{d t}+\left(\frac{\partial L(p)}{\partial p} \cdot \frac{d p}{d t}+R_{c}\right) \cdot i=u \\
f=\frac{1}{2} \cdot \frac{\partial L(p)}{\partial p} \cdot i^{2}
\end{gathered}
$$

where $R_{C}$ represents active resistance and $L(p)$ inductance of EVA coil:

$$
L(p)=L_{0} \cdot \frac{p_{0}}{d+p}
$$

Quantities $p, i$, and $u$ represent the displacement of the LCE with respect to the unmoving support, EVA current, and EVA voltage, respectively. The bronze disk with thickness $d$ doesn't permit inductor to form a complete magnetic circuit of iron; in other words, it inhibits "gluing" of armature and inductor, which is undesirable. In the real cases is $d<<p_{0}$. Mechanical force $f$ produced by the electromagnetic action of EVA coil acts on its armature which is a constituent part of LCE. If the short excitation current pulse for EVA is synchronized with the instant when the armature is passing through the equilibrium position, i.e. when $p=p_{0}$, inductance $L(p)$ can be represented, with sufficient accuracy, as $L(p) \approx L_{0}$. Now, equation (3) in the vicinity of the operating point can be represented as:

$$
L_{0} \cdot \frac{d i}{d t}+R_{c}^{\prime} \cdot i=u
$$

Parameter $R^{\prime}$ represents the equivalent resistance which is dependent on the speed of displacing of the LCE and gradient of the coil inductance at the operating point, given by relation:

$$
R_{c}^{\prime}=R_{c}+\left.\frac{d p}{d t} \cdot \frac{\partial L}{\partial p}\right|_{p=p_{0}}
$$

Taking into account that the excitation current pulses of EVA are short (of the order of several ms), it can be assumed that the equivalent time constant $L_{0} / R_{C}^{\prime}$ is much longer than their duration. On the basis of this, it can be concluded that $R_{c}^{\prime} \cdot i<<u$. Therefore, member $R_{c}^{\prime} \cdot i$ in equation (6) can be neglected. Finally, the approximation of equation (6) is given by:

$$
\frac{d i}{d t}=\frac{u}{L_{0}}
$$

As already mentioned, the pulse excitation is, from the energy point of view, the most suitable method of excitation of electromagnetic resonant conveyors [8]. Owing to the predominantly inductive nature of EVA coil, indicated by equation (8), it is very simple, by applying a suitable control, to generate current pulses in this coil which are of the form of triangular half-waves. From the spectral point of view, a triangular current excitation is characterized by a high harmonic content (the fundamental harmonic $\omega_{0}$ plus higher harmonics at frequencies $\left.2 \omega_{0}, 3 \omega_{0}, \ldots\right)$. In view of equation (4), the excitation force is proportional to the square current, 


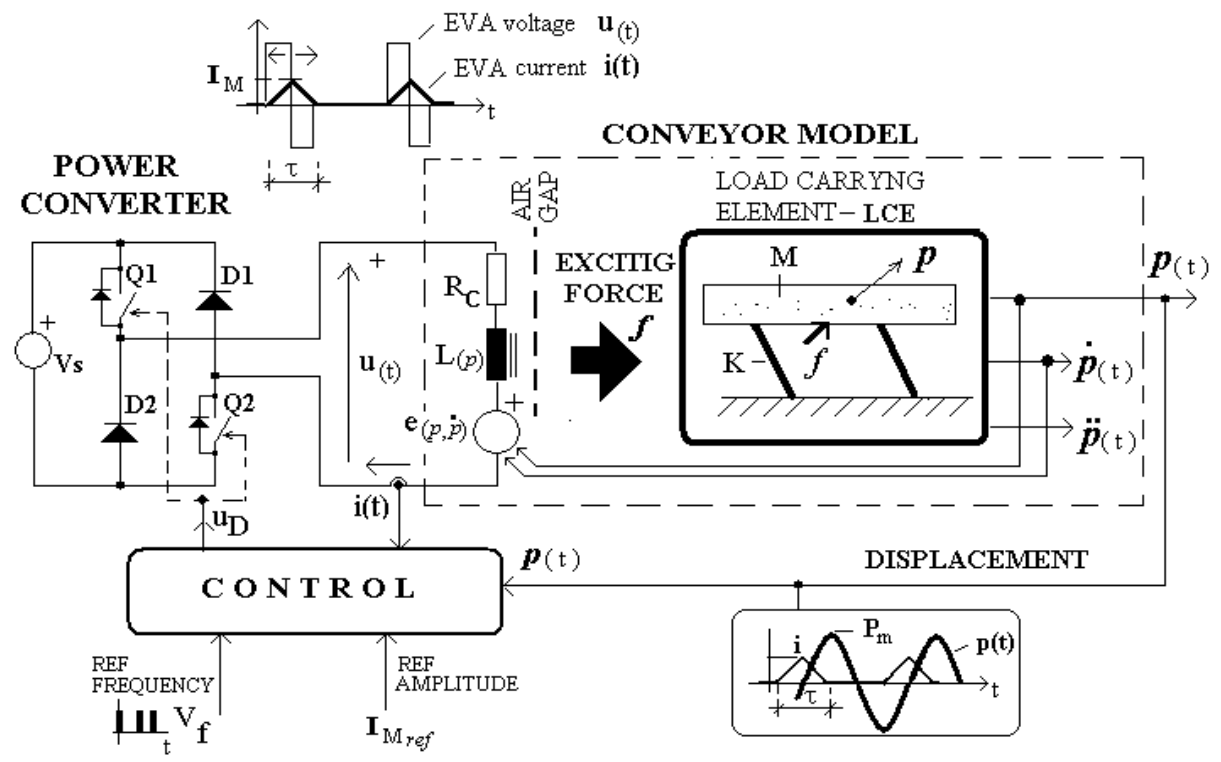

Fig. 6. Block diagram of the amplitude-frequency control of the resonant vibratory conveyor

i.e. $f=k \cdot i^{2}$, and it is concluded that it will contain the same harmonic spectrum. However, irrespective of the pulse excitation of LCE, the output displacement $-p$ is a "smooth" sinusoid, as a consequence of the character of the mechanical part of the system, which, according to equation (2), attenuates higher harmonics for frequencies $\omega>\omega_{0}$, as shown in [7].

The relationship between the pulse duration and the amplitude increase/decrease is highly nonlinear. As the duration of current pulses - $\tau$ in practical applications is very small compared to the cycling period, its contribution can be approximated with a Dirac pulse. According to [15] the strength of the force pulse can be calculated as follows:

$$
A_{f}=\int_{0}^{\tau} f \cdot d t=k \int_{0}^{\tau} i^{2} \cdot d t=k \cdot A_{i}
$$

where $A_{i}$ represent the strength of excitation current pulses and coefficient $k=\frac{1}{2} \cdot \frac{\partial L\left(p_{0}\right)}{\partial p_{0}}$.

To achieve the higher efficiency, it is necessary to generate EVA current pulses at the appropriate moments, so that the amount of energy transferred to the mechanical system, for the given force strength (9), be maximal. This amount of energy can be expressed as:

$$
\Delta E=\int f \cdot d p=\int f \cdot \dot{p} \cdot d t \approx v \cdot \int f \cdot d t=\dot{p} \cdot A_{f}
$$

From this equation it is clear that the current pulse to be generated at the moment when LCE velocity $\dot{p}$ takes the maximum value i.e. when $p=p_{0}$.

According to Fig. 6, voltage of EVA coil is determined by the control signal $u_{D}$. Thus, voltage across EVA excitation coil can be represented by function [15]:

$$
u=\left\{\begin{array}{c}
V_{s}, u_{D}=1 \vee i<0 \\
-V_{s}, u_{D}=0 \wedge i>0 \\
0, u_{D}=0 \wedge i=0
\end{array}\right.
$$

In equation (11) $V_{s}$ denotes voltage of the DC power supply. As a result of tuning the width of the control voltage $u_{D}$ at the output of the bridge converter, a triangular waveform of the current fed to EVA coil is generated. It will be shown later that the current pulse is generated in the way that the current pulse peak corresponds at the instant when the armature passes through the equilibrium position, i.e. when $p=p_{0}$, therefore the inductance of EVA coil in this case is $L(p)=L_{0}$.

From what has been said so far, it can be concluded that the control circuit of Fig. 6 can be realized by introducing feedback in terms of EVA current. In this way it is possible to apply a programmed control of the current pulse strength $A_{i}$ by control of the maximum value of EVA current exciting the vibratory conveyor.

\section{CURRENT CONTROL OF THE ELECTROMAGNETIC VIBRATORY ACTUATOR}

The strategy of current control by means of a switching converter is generally known in power electronics applications of power supplies [16]-[18]. Similar principle has been used for EVA excitation since it represents, as already shown, a predominantly inductive load. The principle diagram of the current control is shown in Fig. 7.

The control circuit which provides control of the amplitude, length, and frequency of the triangular current half-wave is shown in Fig. 7(a). The characteristic waveforms are shown in 


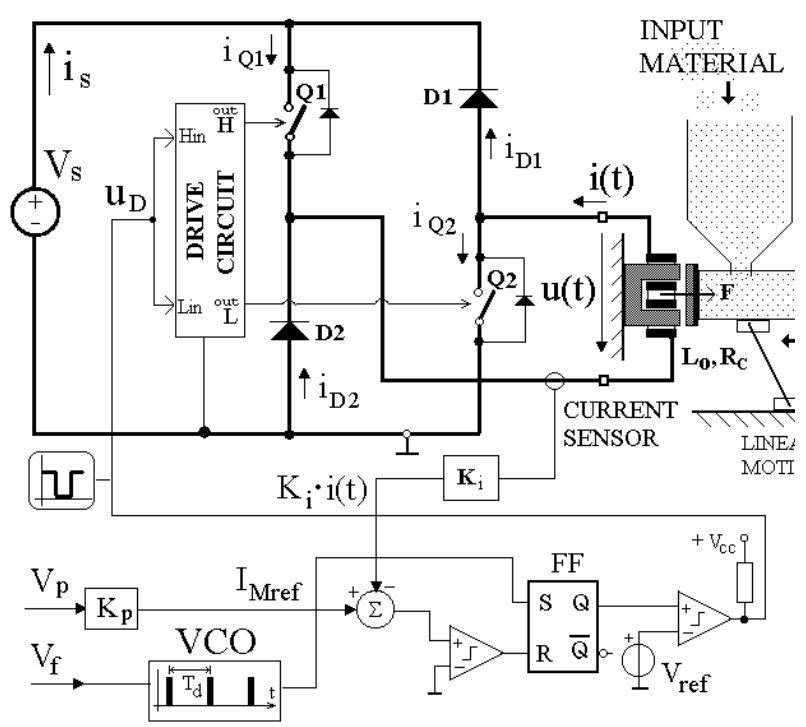

(a)

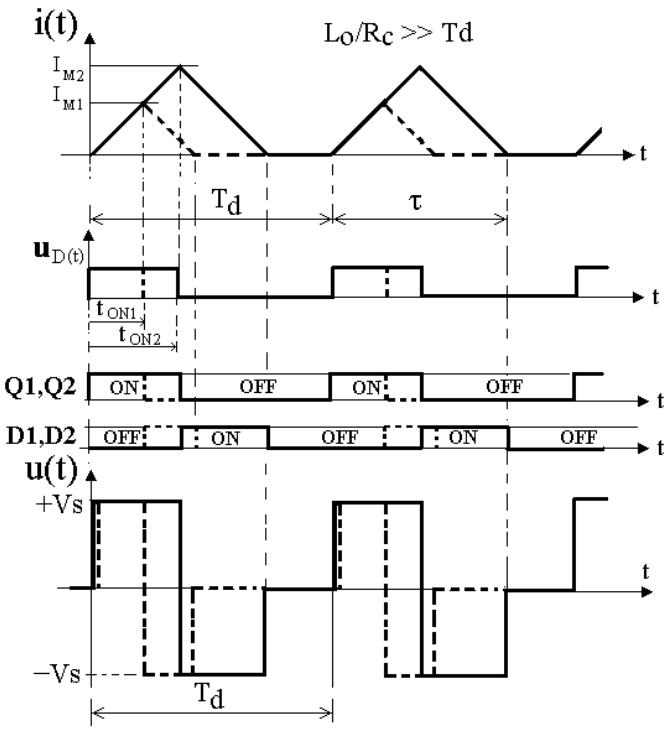

(b)

Fig. 7. The circuit which provides EVA current control. (a) The principle circuit diagram and (b) The characteristics waveforms.

Fig. 7(b). Control of the current pulse strength $A_{i}=\int i^{2} \cdot d t$ is accomplished by a current control loop. Instantaneous value of the actuator current $i(t)$ is measured by the corresponding current sensor. The measured signal is amplified by factor $K_{i}$ and the amplified signal is fed to an adder where it is compared with the reference value $I_{M r e f}$ of the amplitude of the current.

Setting the RS flip-flop FF is accomplished from a voltage controlled oscillator. By feeding signal from the oscillator to $\mathrm{S}$ input of flip-flop FF, the state of logical " 1 " is established at its output $Q$ (output $\bar{Q}$ is at the state of logical “0”), i.e. switches $Q_{1}$ and $Q_{2}$ are turned on simultaneously.

This establishes the current through EVA coil in the form a growing ramp since voltage $+V_{s}$, is applied across the coil terminals. The growth continues until the instantaneous current value reaches the reference value $I_{M r e f}$, when the RESET pulse is generated at R input of flip-flop FF.

The state of logical "0" is established at its output $Q$. As this state is established, switches $Q_{1}$ and $Q_{2}$ are turned off simultaneously, and current of the coil is taken over by return diodes $D_{1}$ and $D_{2}$. EVA current decreases linearly since voltage $-V_{s}$ is applied at the coil terminals. The state of reset is maintained until a new SET pulse arrives from the oscillator. The amplitude and duration of the current are determined by the instant when instantaneous value of the current reaches value $I_{M r e f}$. Since inductive resistance predominates, the rise time is approximately the same as the fall-off time, given by relation:

$$
\tau_{r} \approx \tau_{f}=\frac{L_{0} \cdot I_{M r e f}}{V_{s}}
$$

i.e. total time duration of the current pulse within one period is given by relation:

$$
\tau \approx \frac{2 \cdot L_{0} \cdot I_{M r e f}}{V_{s}}
$$

Based on equations (9) and (13) one obtains an expression for current strength $A_{i}$ :

$$
\begin{gathered}
A_{i}=2 \cdot \int_{0}^{\tau}\left(2 I_{M} \cdot \frac{t}{\tau}\right)^{2} \cdot d t=\frac{1}{3} \cdot I_{M r e f}^{2} \cdot \tau \\
A_{i}=\frac{2}{3} \cdot I_{M r e f}^{3} \cdot \frac{L_{0}}{V_{s}}
\end{gathered}
$$

Change in the amplitude of oscillations in the resonant mode at frequency $\omega_{0}$ is given by a relation:

$$
\Delta p=K_{p} \cdot k \cdot \omega_{0} \cdot A_{i}
$$

From these relations it follows that the time duration of the current half-wave is linear function of $I_{\text {Mref }}$. This means that tuning the amplitude of vibrations of the LCE is accomplished by tuning the value of $I_{\text {Mref }}^{3}$. As already mentioned, control of the frequency of vibrations is accomplished by tuning the frequency of the pulses setting flip-flop FF.

\section{Simulation RESUltS}

This section presents the results obtained by the simulation model based on equations (2)-(5). The basic mechanical parameters of the simulation are: $m_{k 0}$ - mass of the moving part of the vibratory conveyor, $k_{e}$ - total stiffness of the elastic elements, and $b_{e}$-damping of the system. On the basis of these parameters the mechanical parameters of the system which appear in the model are calculated: static gain 
TABLE I

SIMULATION PARAMETERS

\begin{tabular}{|l|c|c|c|c|c|}
\hline \multicolumn{4}{|c|}{$\begin{array}{l}\text { MECHANICAL } \\
\text { PARAMETERS }\end{array}$} & \multicolumn{2}{c|}{$\begin{array}{c}\text { ELECTRICAL } \\
\text { PARAMETERS }\end{array}$} \\
\hline $\begin{array}{c}m_{k 0} \\
{[\mathrm{~kg}]}\end{array}$ & 1.15 & $\begin{array}{c}\omega_{0} \\
{[\mathrm{rad} / \mathrm{s}]}\end{array}$ & 314.56 & $\begin{array}{c}R_{C} \\
{[\Omega]}\end{array}$ & 100 \\
\hline $\begin{array}{c}p_{0} \\
{[\mathrm{~mm}]}\end{array}$ & 5.00 & $\begin{array}{c}K_{p} \\
{[\mathrm{~mm} / \mathrm{N}]}\end{array}$ & 0.0088 & $\begin{array}{c}L_{0} \\
{[\mathrm{H}]}\end{array}$ & 1.3 \\
\hline $\begin{array}{l}d \\
{[\mathrm{~mm}]}\end{array}$ & 0.05 & $\xi$ & 0.0095 & $\begin{array}{c}V_{s} \\
{[\mathrm{~V}]}\end{array}$ & 400 \\
\hline
\end{tabular}

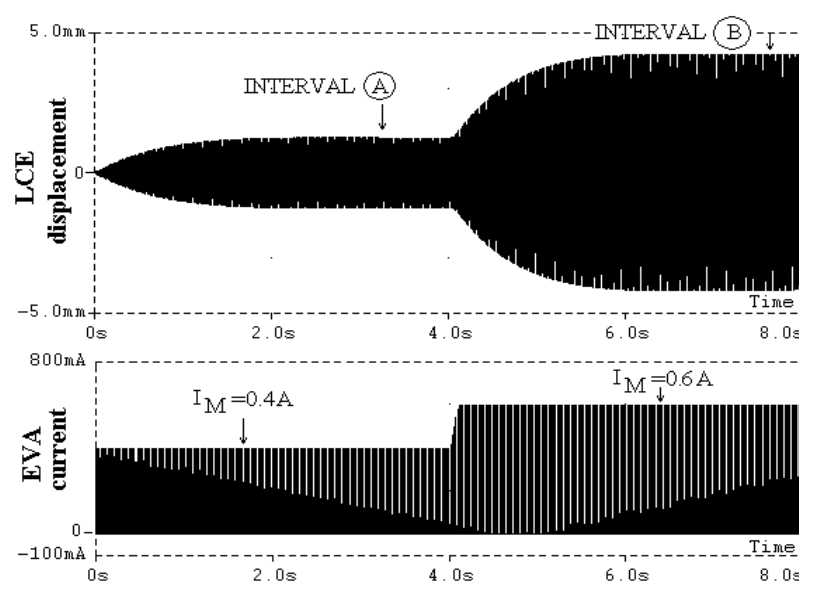

Fig. 8. Adjustment of the amplitude oscillations of LCE by means of EVA current.

- $K_{p}=1 / k_{e}$ and damping factor $\xi=b_{e} / 2 \omega_{0} m_{k 0}$. Resonant frequency of the system was $\omega_{0}=314.56 \mathrm{rad} / \mathrm{s}$. The electrical parameters of the simulation are: $R_{c}$ - active resistance of EVA coil, $L_{0}-$ inductance of EVA coil, and $V_{s}$ - power supply voltage. Real parameter values used in the simulation are presented in Table I.

In the simulation, the amplitude, time duration, and frequency of the excitation current of EVA coil have been selected for control variables. The simulation results obtained for the case amplitude control, at the established resonant mode with the excitation frequency $50 \mathrm{~Hz}$, are shown in Fig. 8 for two amplitude values of the excitation current $\left(I_{M 1}=0.4 \mathrm{~A}\right.$ and $\left.I_{M 2}=0.6 \mathrm{~A}\right)$.

Fig. 9 shows in detail intervals A and B marked in Fig. 8. Fig. 9(a) shows the case when amplitude of the excitation current is adjusted to value $I_{M(A)}=0.4 A$ and its duration to $\tau_{A}=2.4 m s$. Under these conditions amplitude of the oscillations was $P_{m(A)}=1.25 \mathrm{~mm}$. Fig 9(b) shows the case when amplitude of the excitation current is adjusted to value

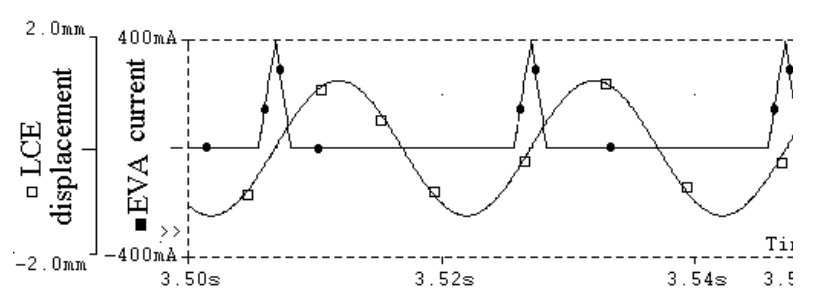

(a)

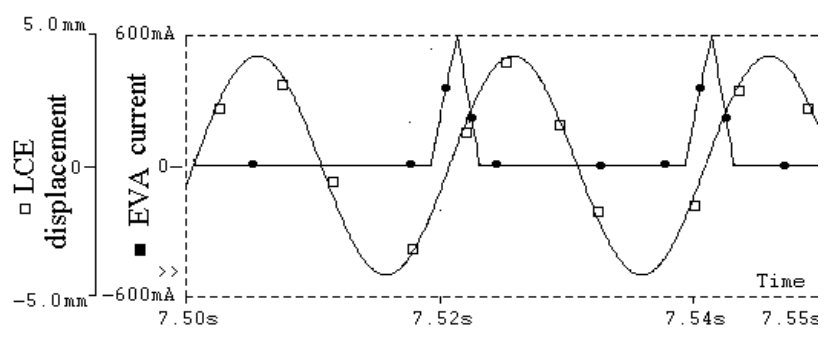

(b)

Fig. 9. Details showing the influence of increased excitation current on LCE displacement for (a) interval A and (b) interval B.

$I_{M(A)}=0.6 A$ and its duration to $\tau_{B}=3.7 \mathrm{~ms}$. Under these conditions amplitude of the oscillations was $P_{m(A)}=4 \mathrm{~mm}$.

Therefore, in this case amplitude of the oscillations is about three times larger (or more accurately 3.2 times) which is in coincidence with the relations (13) and (15).

On the basis of the obtained waveforms, one concludes that under these conditions the excitation pulse approximately coincides with the instant the output displacement $-p$ passes through the equilibrium position and that the amplitude of LCE oscillations is influenced by its duration.

By adjusting the amplitude and duration of the excitation current, it is possible to tune the intensity of LCE oscillations of a vibratory conveyor. In both cases, the maximum of the excitation current and zero displacement are in phase, which was to be expected since operation in the resonant mode was maintained.

On the basis of the obtained waveforms, one concludes that under these conditions the excitation pulse approximately coincides with the instant the output displacement $-p$ passes through the equilibrium position and that the amplitude of LCE oscillations is influenced by its duration. By adjusting the amplitude and duration of the excitation current, it is possible to tune the intensity of LCE oscillations of a vibratory conveyor. In both cases, the maximum of the excitation current and zero displacement are in phase, which was to be expected since operation in the resonant mode was maintained.

Then, the simulation of the frequency control has been performed assuming that load of the conveyor is increased. The simulation results are shown in Fig. 10. An interval of 10s was observed. Until to instant $t=2 s$ resonant mode with empty vibratory trough was established. The initial mass of the moving part, consisting of the trough support, trough itself, and 

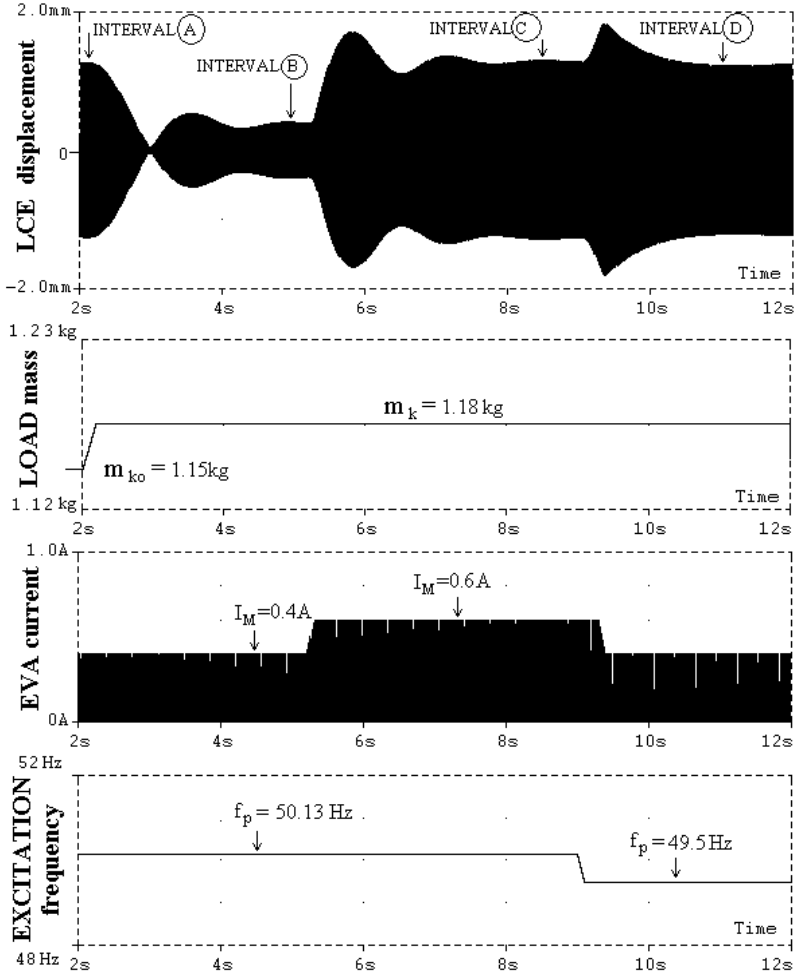

Fig. 10. The characteristic waveforms of resonant conveyors when mass of the load is increased.

moving armature, was $m_{k o}=1.15 \mathrm{~kg}$. During interval $A$, the amplitude of oscillations of the system was $P_{m(A)}=1.25 \mathrm{~mm}$. The excitation frequency was tuned to the resonant frequency of the mechanical system $50.13 \mathrm{~Hz}$. Amplitude of the excitation current was set to value $I_{M(A)}=0.4 A$ and its duration to $\tau_{A}=2.4 m s$. Fig. 11(a) presents this interval in detail. At the instant immediately after $t=2 s$, an abrupt increase of the vibratory troughl load of $30 \mathrm{~g}$ was set to occur, i.e. $m_{k}=1.18 \mathrm{~kg}$. A significant reduction of the amplitude followed and through a transient process lasting approximately 3s, a new steady state was established (interval $B$ ). The amplitude stabilized at value $P_{m(B)}=0.38 \mathrm{~mm}$.

The excitation frequency remained the same, but due to the change of the load mass, resonant frequency of the mechanical part of the system changed to value $49.5 \mathrm{~Hz}$. So there, was a mismatch between the excitation and resonant frequencies. The amplitude and time duration of the excitation current remained unchanged compared to those of interval A. Fig. 11(b) shows this interval in detail. It can be noticed that a significant phase shift exists between the maximum of the excitation current pulse and zero value of the sinusoidal displacement $-p$ of LCE. If an increase of the amplitude of oscillations is to be achieved, it is necessary to increase the amplitude and duration of the coil current. This event is assumed to have occurred at instant $t=5 \mathrm{~s}$. Amplitude of the excitation current is adjusted to

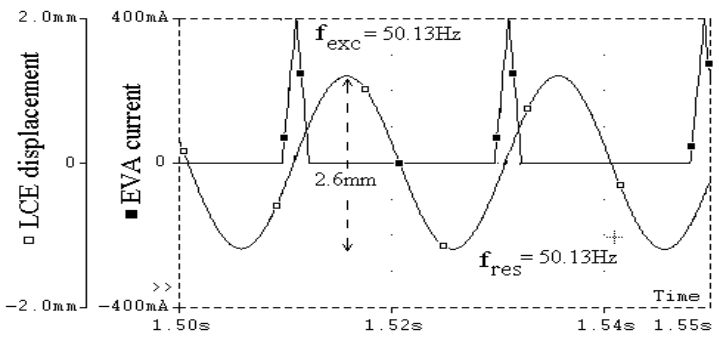

(a)

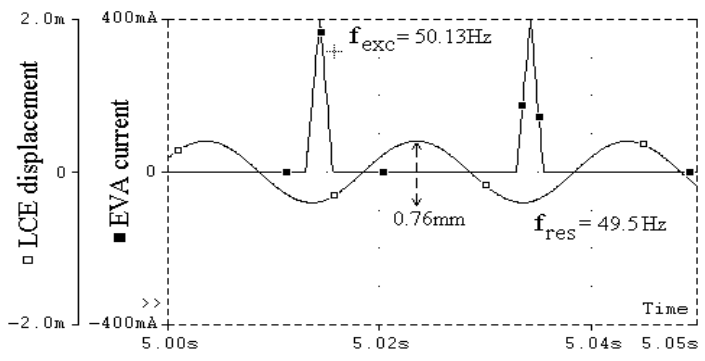

(b)

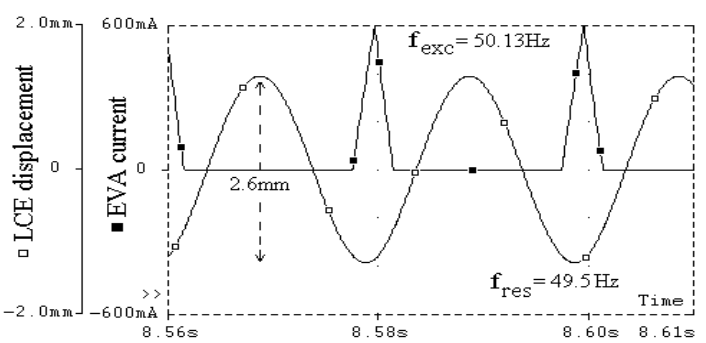

(c)

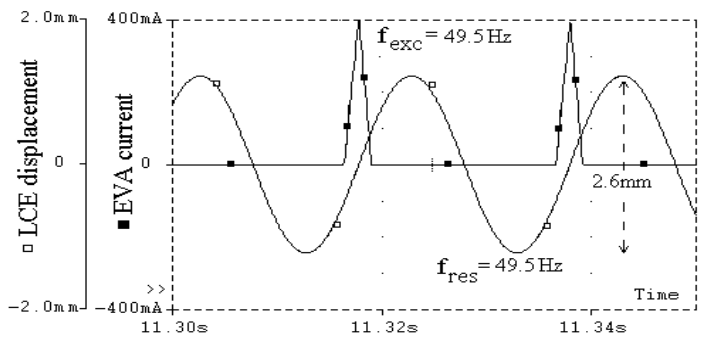

(d)

Fig. 11. Details showing the influence of increased mass of the vibratory trough of a conveyor for (a) interval A, (b) interval B, (c) interval C and (d) interval D.

value $I_{M(C)}=0.6 \mathrm{~A}$ and its time duration to $\tau_{C}=4 m s$, as shown in Fig. 11(c).

The increase of the amplitude of oscillations compared to that of interval $\mathrm{B}$ is possible to achieve by retaining the same values of the amplitude and duration of the excitation current as those of interval $B$ and by tuning the frequency of the excitation current pulse to the new mechanical resonance frequency $49.5 \mathrm{~Hz}$. Namely, by tuning the frequency of the excitation current signal to value $49.5 \mathrm{~Hz}$ at instant $\mathrm{t}=9 \mathrm{~s}$, maintaining the amplitude of the oscillations $P_{m(D)}=1.25 \mathrm{~mm}$ has been accomplished through a transient 


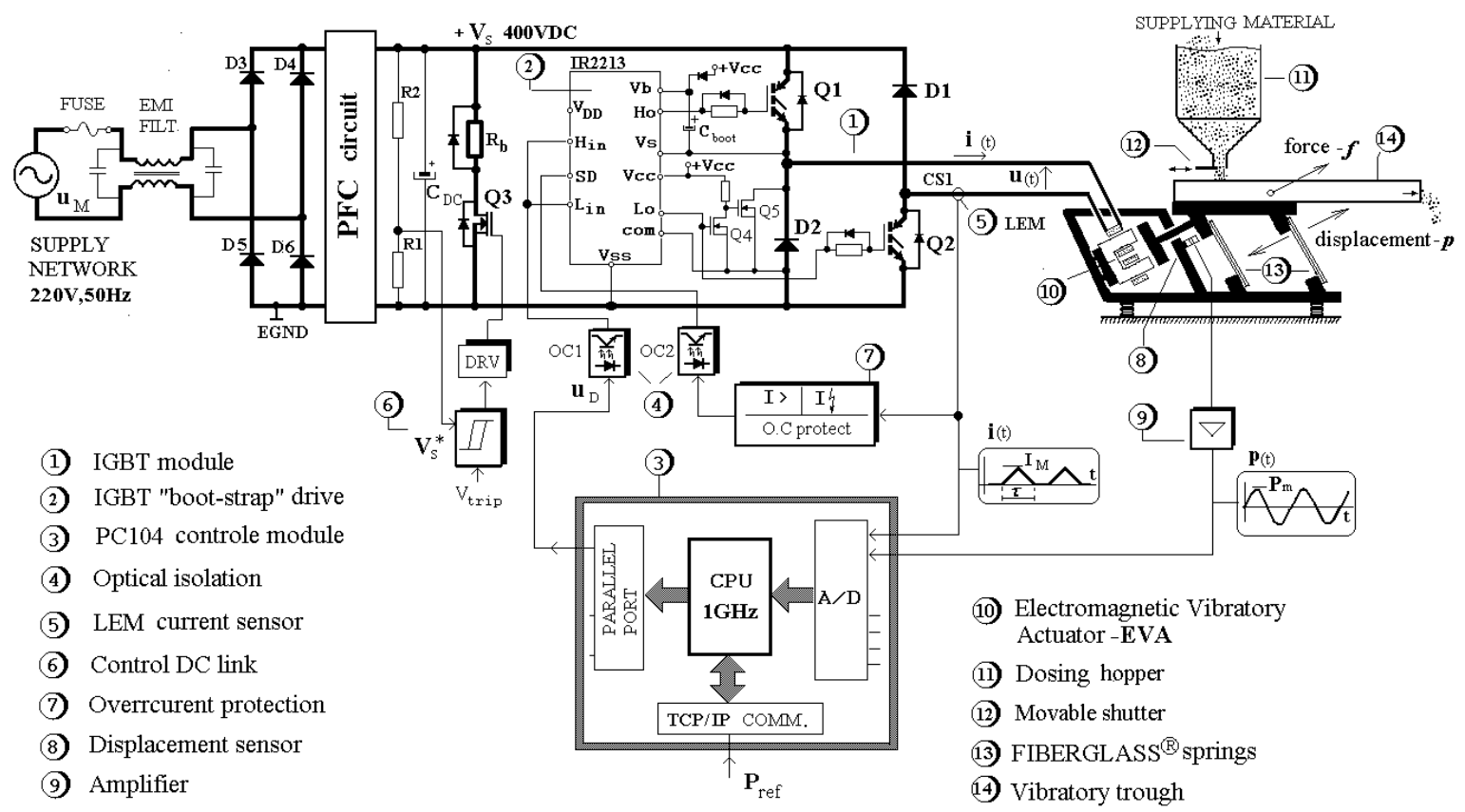

Fig. 12. Block diagram of the realized current controller of the electromagnetic vibratory conveyor.

process lasting 3s. This steady state is established in interval D, shown in detail in Fig. 11(d).

\section{PRACTICAL REALIZATION}

A practically realized IGBT transistor converter for excitation of a vibratory conveyor having electromagnetic excitation is described in this section. Fig. 12 shows block diagram of the complete system.

The transistor converter for EVA excitation consists of an input full-wave rectifier comprising diodes D3-D6 and an output converter which serves for generation of the pulsating dc excitation current. In order to eliminate the noise generated by the converter, an EMI filter is inserted towards the network $220 \mathrm{~V}, 50 \mathrm{~Hz}$.

In addition to the diode rectifier, the input AC/DC converter contains a circuit for power factor correction having at its output dc voltage $400 \mathrm{~V}$. The output converter for excitation of EVA coil, realized by using the asymmetric half-bridge - (1), consists of two IGBT transistors $\mathrm{Q}_{1}$ and $\mathrm{Q}_{2}$ positioned in one diagonal of the bridge and two return diodes $D_{1}$ and $D_{2}$ positioned in the other diagonal. Excitation of the IGBT's is accomplished by the driving circuit - (2) which contains two independent channels for driving the upper and lower transistors. Driving the upper transistor is realized by a „floating” circuit which can sustain high voltage and is immune to sharp voltage edges ( $d v / d t)$. The control part - (3) is based on industrial PC104 module where the algorithms for search and tracking of the resonant frequency are implemented, together with current control, tuning amplitude of the oscillations, etc. The controller as well as the circuit for monitoring the voltage if the intermediate DC circuit are galvanic isolated by opto-couplers - (4) from the power part of the converter.

The value of EVA current is measured by a Hall Effect current sensor, the so called LEM current sensor - (5). Discharge of the electrolytic capacitors in the intermediate DC circuit is carried out via resistor $\mathrm{R}_{\mathrm{b}}$ and transistor $\mathrm{Q}_{3}$ which is controlled by the voltage monitor - (6) containing a built-in hysteresis, which „observes“ voltage of the DC circuit $\mathrm{V}_{\mathrm{s}}$ and compares it with the set threshold $V_{\text {trip }}$. The over-current and over-load protection circuit is realized by the current protection - (7) block. The „intervening“ system of protection is applied. Under normal conditions, the load current is programmed by the controller. In the case of a direct short circuit or overload, block (7) takes over. Measurement of the output displacement of the LCE and detection of its passage through the equilibrium position is accomplished by a non-contact inductive displacement sensor - (8), operating in the displacement range $\pm 5 \mathrm{~mm}$ and frequency range $0-1 \mathrm{kHz}$. The signal of this sensor is transmitted by an electronic amplifier - (9) and normalized to the $0-5 \mathrm{~V}$ level.

\section{THE EXPERIMENTAL RESULTS}

The experimental results are obtained with a realized laboratory prototype of the resonant vibratory conveyor having 


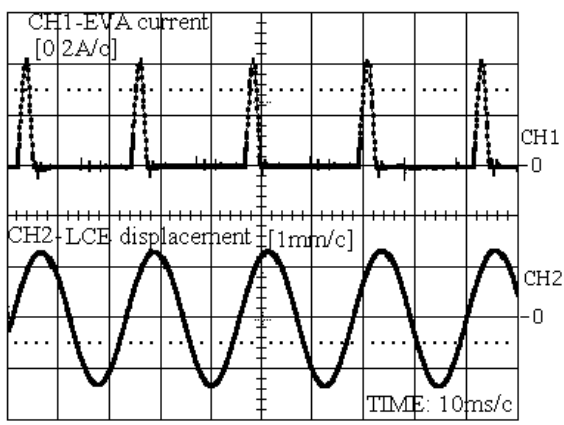

(a)

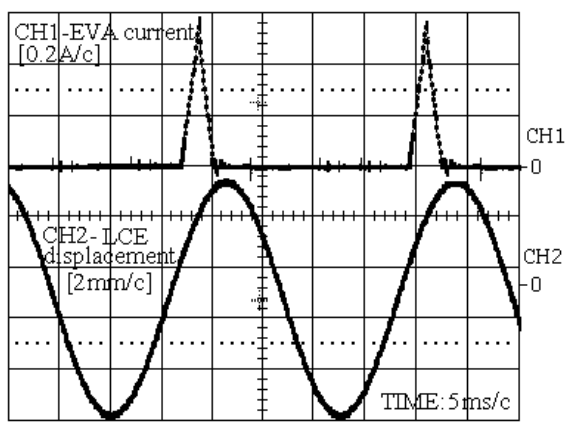

(b)

Fig. 13. Influence of the current pulse strength $A i$ on the output displacement of the vibratory trough for (a) amplitude of current $\mathrm{I}_{\mathrm{M}}=0.4 \mathrm{~A}$ and pulse duration $\tau=2.5 \mathrm{~ms}$ and (b) amplitude of current $\mathrm{I}_{\mathrm{M}}=0.6 \mathrm{~A}$ and pulse duration $\tau=3.8 \mathrm{~ms}$.

electromagnetic excitation, where the amplitude-frequency control has been applied. In the experiments the measured quantities of interest were: EVA current- $i(t)$ and displacement of LCE $-p(t)$.

The influence of variations of the amplitude and duration of the current pulses on the displacement of the LCE, at resonant frequency $45.5 \mathrm{~Hz}$ is shown in Fig. 13.

Fig. 13(a) presents the oscilloscope records of EVA current and LCE displacement for the reference value of the current $I_{\text {Mref } 1}=0.4 \mathrm{~A}$. Under these conditions, time duration of the current pulses was approximately $\tau_{1}=2.5 \mathrm{~ms}$, while the accomplished amplitude of the output displacement of LCE was $P_{m 1}=1.3 \mathrm{~mm}$. Integral action of the current pulse is a measure of the excitation force acting on LCE. By this integral action of the current pulse one takes into account its amplitude and its duration. In this case current pulse strength is $A_{i 1} \approx 0.133\left[A^{2} m s\right]$. Fig. 13(b) shows the records of EVA current and LCE displacement for the reference value of the current $I_{\text {Mref 2 }}=0.6 \mathrm{~A}$. Under these conditions, duration of the current pulses was approximately $\tau_{2}=3.8 \mathrm{~ms}$, while the accomplished amplitude of the output displacement of LCE

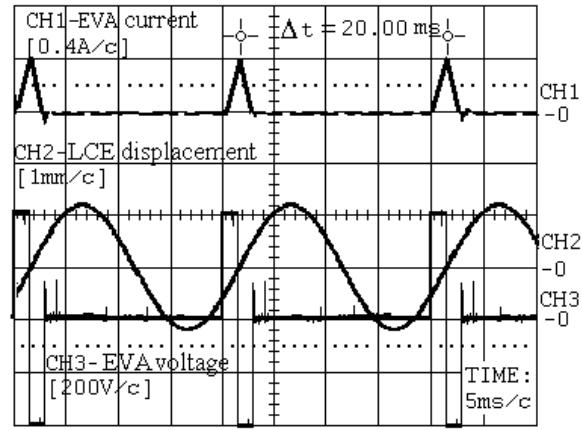

(a)

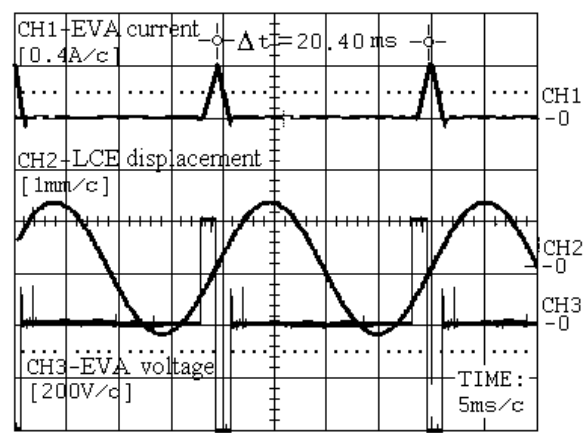

(b)

Fig. 14. Compensation of the influence of load change by the change of frequency of the EVA excitation current. (a) The frequency of EVA excitation current $50.13 \mathrm{~Hz}$ and (b) frequency of EVA excitation current $49 \mathrm{~Hz}$

was $P_{m 2}=4.1 \mathrm{~mm}$. In this case the current pulse strength is $A_{i 2} \approx 0.456\left[A^{2} m s\right]$. With a constant LCE load (i.e. no change of the resonant mode) and 50\% change of the current pulses amplitude, almost 3.4 times higher current pulse strength, i.e. amplitude of the oscillations, is obtained. The obtained experimental result is in agreement with the simulation result shown in Fig. 9.

Fig. 14 shows the compensation of the change caused by a change of LCE load. Fig. 14(a) shows the characteristic waveforms of the EVA excitation current and output displacement of LCE for the load mass $m_{k 0}=1.15 \mathrm{~kg}$, giving resonant frequency $50.13 \mathrm{~Hz}$. Fig. 14(b) gives the same waveforms, for the load mass increased by $50 \mathrm{~g}$, i.e. for load mass $m_{k 1}=1.20 \mathrm{~kg}$ (the corresponding resonant frequency is $49 \mathrm{~Hz})$.

Fig. 15 shows the comparative results for controlled vibratory conveying drives with switch mode and conventional SCR converters for the case when the resonant frequency deviates from the nominal value $(50 \mathrm{~Hz})$ for only 1 $\mathrm{Hz}$ i.e. whose value is $49 \mathrm{~Hz}$. Since the SCR converter operation can not keep tracking the resonant frequency, there 


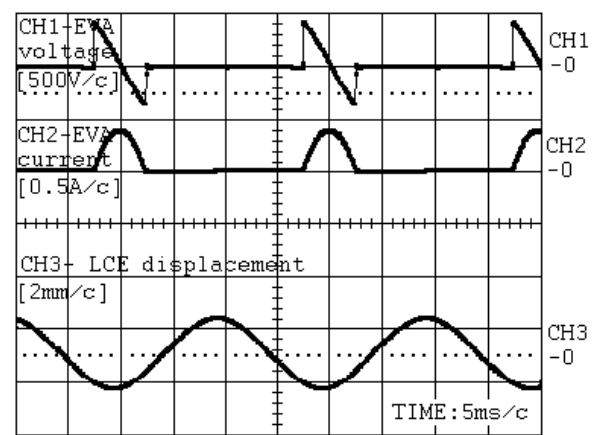

(a)

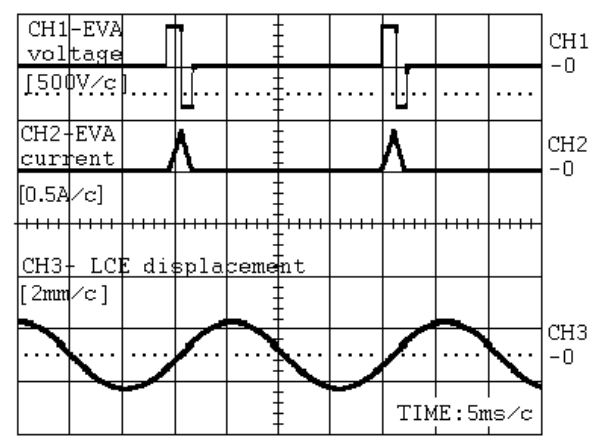

(b)

Fig. 15. Comparison efficiency of regulated vibratory conveying drives for resonant frequency $49 \mathrm{~Hz}$. (a) SCR power converter-frequency of EVA excitation current $50 \mathrm{~Hz}$ and (b) The switch mode power converter-frequency of EVA excitation current $49 \mathrm{~Hz}$.

is a significant deterioration in the efficiency of the drive.

As can be seen from the oscilloscopic records in Fig. 15(a), in the case of the operation with SCR converter, the moment of reaching maximum value of current impulse EVA is phase-shifted significantly from the moment when the armature passing through the equilibrium position i.e. $p=p_{0}$. In that case there is a significant deviation from the optimal regime that is mentioned in section III. To maintain a constant amplitude vibration of LCE, strength of current pulses- $A_{i}$ (i.e. effective value of EVA current) is significantly increased. As a result there are increased copper-losses in the EVA coil.

Switch-mode power converter with proposed current control allows tracking resonant frequency and thus provides a better efficiency. In that case the current pulses of EVA are generated at the optimal moment, so that the effective value of current pulses i.e. strength of excitation current pulses- $A_{i}$ is significantly lower, as shown in Figure 15 (b). This is particularly true in cases where the damping factor $\xi$ is relatively small, which is very often in practice. Previous results were obtained for the damping factor $\xi=0.0085$. In that case a sharp resonance peak of the transmission characteristics for the complete resonant vibratory conveying drive is obtained.

\section{CONCLUSIONS}

The paper presents a possible solution for the amplitude-frequency control of the resonant vibratory conveyor having electromagnetic excitation. The solution represents a considerable improvement compared to the conventional, phase controlled thyristor and triac drives. The basic shortcoming of the phase control is that it can only tune amplitude of the oscillations of LCE but not their frequency. The proposed EVA current control is based on the switching topology. This topology offers numerous advantages with respect to the conventional thyristor topology. The most significant advantage is the possibility of independent tuning of the amplitude and frequency of oscillations of the conveyor's LCE. As a result, it is now possible to track the resonant mode of a vibratory conveyance system with sufficient speed and precision.

The presented simulations and experimental results have shown that EVA current control resulted in a very efficient method for both amplitude and frequency control, which is essential for tuning the speed of conveyance of granular materials, and for frequency control, which maintains the resonant mode of the vibratory conveyor. The resonant mode is very important from the point of view of energy efficiency and minimization of energy consumption of the whole vibratory-conveyance drive.

By current control it has been achieved that a vibratory-conveyance system became a controllable mechanical oscillator, whose operation is independent of the supply frequency.

\section{ACKNOWLEDGMENT}

The authors gratefully acknowledge the constructive comments and valuable suggestions of anonymous reviewers. This investigation has been carried out with the financial support of the Serbian Ministry of Science- project No: TR33022.

\section{REFERENCES}

[1] I.F. Goncharevich, K.V. Frolov, and E.I. Rivin, Theory of vibratory technology, Hemisphere Publishing Corporation, New York, 1990.

[2] T. Dyr and P. Wodzinski, "Model particle velocity on a vibrating surface," Physicochemical Problems of Mineral Processing, Vol. 36, pp. 147-157, May 2002.

[3] E. M. Sloot and N. P. Kruyt, "Theoretical and experimental study of the conveyance of granular materials by inclined vibratory conveyors," Powder Technology, Vol. 87, No. 3, pp. 203-210, 1996. 
[4] G. R Soto-Yarritu and A. A Martinez, "Computer simulation of granular material: vibrating feeders," Powder Handling and Processing, Vol. 13, No. 2, 2001.

[5] E. H. Werninck, Electric Motor Handbook, McGraw-HILL Book Company (UK) Limited, 1978.

[6] M. A. Parameswaran and S. Ganapahy, "Vibratory conveying-analysis and design: A review," Mechanism and Machine Theory, Vol. 14, No. 2, pp. 89-97, Apr. 1979.

[7] T. Doi, K. Yoshida, Y. Tamai, K. Kono, K. Naito, and T. Ono, "Modelling and feedback control for vibratory feeder of electromagnetic type," Journal of Robotics and Mechatronics, Vol. 11, No. 5, pp. 563-572, Jun. 1999.

[8] T. Doi, K. Yoshida, Y. Tamai, K. Kono, K. Naito, and T. Ono, "Feedback control for vibratory feeder of electromagnetic type,” Proc. ICAM’98, pp. 849-854, 1998.

[9] Z. Despotovic and Z. Stojiljkovic, "Power converter control circuits for two-mass vibratory conveying system with electromagnetic drive: Simulations and experimental results," IEEE Trans. Ind. Electron., Vol. 54, No. 1, pp.453-466, Feb. 2007.

[10] I. J. Sokolov, V. I. Babitsky, and N. A. Halliwell, "Autoresonant vibro-impact system with electromagnetic excitation,” Journal of Sound and Vibration, No. 308, pp. 375-391, 2007.

[11] S. Seely, Electromechanical energy conversion”, McGraw-HILL Book Company INC., New York, 1962.

[12] Z. Despotovic, and Z. Stojiljkovic, "PSPICE simulation of two mass vibratory conveying system with electromagnetic drive," PROCEEDINGS of International conference "Computer as a tool", Vol. 2, pp. 1509-1512, 2005.

[13] G. Winkler, "Analysing the vibrating conveyor," International Journal of Mechanics, Vol. 20, pp. 561-570, 1978.

[14] P. Wolfsteiner and F. Pfeiffer, "Dynamics of a vibratory feeder," proceedings of detc '97, asme design engineering technical conferences, DETC97/VIB-3905, pp. 1-9, 1997.

[15] A. I. Ribic and Z. Despotovic, "High-performance feedback control of electromagnetic vibratory feeder," IEEE Trans. Ind. Electron., Vol. 57, No. 9, pp.3087-3094, Aug. 2010.

[16] N. Mohan, T. M. Undeland, and W. P. Robbins, Power Electronics-Converters, Applications and Design, John Wiley\&Sons, INC., New York, 1995.

[17] M. H. Rashid, Power Electronics, Prentice-Hall Englewood Cliffs, New York, 1988.

[18] K. Thorborg, Power Electronics, Prentice Hall International (UK) Ltd. 1988.

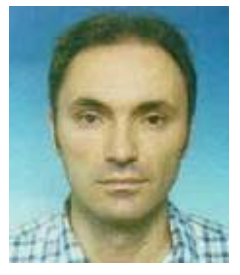

Željko V. Despotović was born in Prijepolje, Serbia, in 1964. He received the B.Sc., M.Sc., and Ph.D. degrees from the Department of Power Converters and Drives, School of Electrical Engineering, University of Belgrade, Belgrade, Serbia, in 1990, 2003, and 2007, respectively. Since 1991, he has been with the Department of Robotics and Mechatronics, Institute "Mihajlo Pupin”, University of Belgrade, where he is currently an Associate Fellow in the Laboratory for Digital Control of Power Converters and Electrical Drives, School of Electrical Engineering. $\mathrm{He}$ is also currently a PhD.E.E-Scientific Fellow and a Senior Research and Development Engineer of power electronics. His research interests include the fields of power electronics, industrial electronics, mechatronics, and vibration control. He is member of the IEEE Industrial Electronics Society, the IEEE Industry Applications Society, and the IEEE Power Electronics Society. He has the graduate engineer's licenses: Responsible Designer of Electrical Drive Control-Automatic, Measuring, and Regulation and Responsible Designer of Low and Medium Voltage Power System, Serbian Chamber of Engineers. He is professor at the High School of Professional Studies in Electrical Engineering and Computer Science - Belgrade, Serbia, since February 2010.

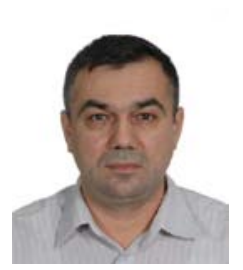

Aleksandar I. Ribić was born in Dubrovnik, Croatia, in 1966. He received the Bs.C. degree from the Faculty of Electrical Engineering Banja Luka, University of Banja Luka, Bosnia and Herzegovina, in 1992, and the M.Sc. and Ph.D. degrees from the School of Electrical Engineering, University of Belgrade, Belgrade, Serbia, in 2003 and 2010 respectively. Since 2004, he has been with the Automatic Control Department of the Institute "Mihajlo Pupin,” University of Belgrade. His research interests include the fields of process and power plant control.

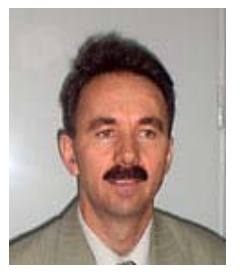

Vladimir M. Šinik was born in Berkovići, Bosnia and Herzegovina, in 1958. He received the B.Sc. and M.Sc. degrees in electrical engineering from the University of Sarajevo-Bosnia and Herzegovina in 1981 and 1991 respectively. In 2001, he joined of the Technical Faculty "Mihajlo Pupin" Zrenjanin- University of Novi Sad, Serbia. He received the Ph.D. degrees of the Technical Faculty "Mihajlo Pupin" Zrenjanin -University of Novi Sad, Serbia. His research interests are in Power Electronics, Power Converters, Uninterruptible Power Supply and Electromagnetic Compatibility. 